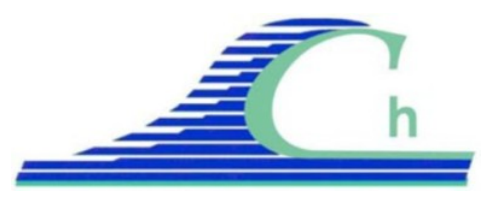

XII ${ }^{\text {ìmes }}$ Journées Nationales Génie Côtier - Génie Civil

Cherbourg, 12-14 juin 2012

DOI:10.5150/jngcgc.2012.042-R @ Editions Paralia CFL

disponible en ligne - http://www.paralia.fr - available online

\title{
Concentration de sédiment en suspension près de la surface en Manche : modélisation numérique vs observation satellite
}

\author{
Aurélie RIVIER ${ }^{1,2}$, Nicolas GUILLOU ${ }^{1}$, \\ Georges CHAPALAIN $^{1}$, Francis GOHIN ${ }^{2}$
}

1. Centre d'Etudes Techniques Maritimes Et Fluviales (CETMEF), Laboratoire de Génie Côtier et Environnement (LGCE), 155 rue Pierre Bouguer, Technopole Brest Iroise BP 5, 29280 Plouzané, France.

2. IFREMER, Centre de Bretagne, ODE/DYNECO/PELAGOS, BP 70, 29280 Plouzané, France.

aurelie.rivier@ifremer.fr

\section{Résumé :}

La présente étude vise à analyser la variabilité spatio-temporelle de la concentration de sédiment en suspension (CSS) en surface en Manche par une approche conjointe d'observation satellite et de modélisation numérique. Les prédictions numériques sont comparées à trois images satellite claires représentant différentes conditions hydrodynamiques de marée et de houle. Le modèle reproduit globalement les structures de CSS observées en surface sur les images satellite autour de l'ile de Wight et dans le golfe normano-breton. Le modèle permet d'analyser la composition granulométrique de la CSS et indique que les particules fines $(<50 \mu \mathrm{m})$ sont majoritaires en surface. Les classes de taille supérieure (entre 100 et $500 \mu \mathrm{m}$ ) ont un impact en surface principalement autour de l'ile de Wight et dans le golfe normano-breton. Une sensibilité au temps d'initialisation (spin up) est mise en avant.

Mots-clés : Modélisation - Satellite - Sédiment en suspension - Marée - Houle

\section{Introduction}

La concentration de sédiment en suspension (CSS) près de la surface est un indicateur de la dynamique côtière et agit sur la clarté des eaux qui est déterminante pour la production biologique du phytoplancton. Les variabilités spatiales et temporelles de ce paramètre en fonction des forçages de marée et de houle ont récemment été mises en évidence par RIVIER et al. (2012) à l'échelle du domaine côtier de la Manche. Basée sur l'analyse statistique d'une série d'images satellite, cette étude a abouti à une cartographie des zones d'influences des régimes de marée sur la variabilité temporelle de la CSS de surface à la résolution du cycle morte-eau/vive-eau (figure 1).

La modélisation numérique hydro-sédimentaire tridimensionnelle (3D) permet d'affiner cette analyse en ajoutant la dimension verticale et en affinant la résolution temporelle par rapport aux images satellite quotidiennes et bidimensionnelles. Les transports en suspension à l'échelle de la Manche ont ainsi fait l'objet de nombreuses études 
numériques confrontées aux observations satellite (e.g., PULS et al., 1994 ; SOUZA et al., 2007 ; DOBRYNIN et al., 2010).

La présente étude complète ces différents travaux en analysant à partir d'un modèle hydro-sédimentaire 3D multiclasse le développement des CSS de surface en Manche en fonction des forçages dominants de marée et de houle. Après une description succincte de la base de données satellite utilisée, du modèle numérique et des conditions de simulation, les résultats du modèle sont comparés à une sélection d'images "claires". Ces comparaisons conduisent à une première analyse de la CSS de surface en Manche suivant les conditions hydrodynamiques. La composition de la CSS de surface est explorée et une étude de sensibilité analyse l'influence du temps d'initialisation ("spinup") sur la qualité ces prédictions numériques.

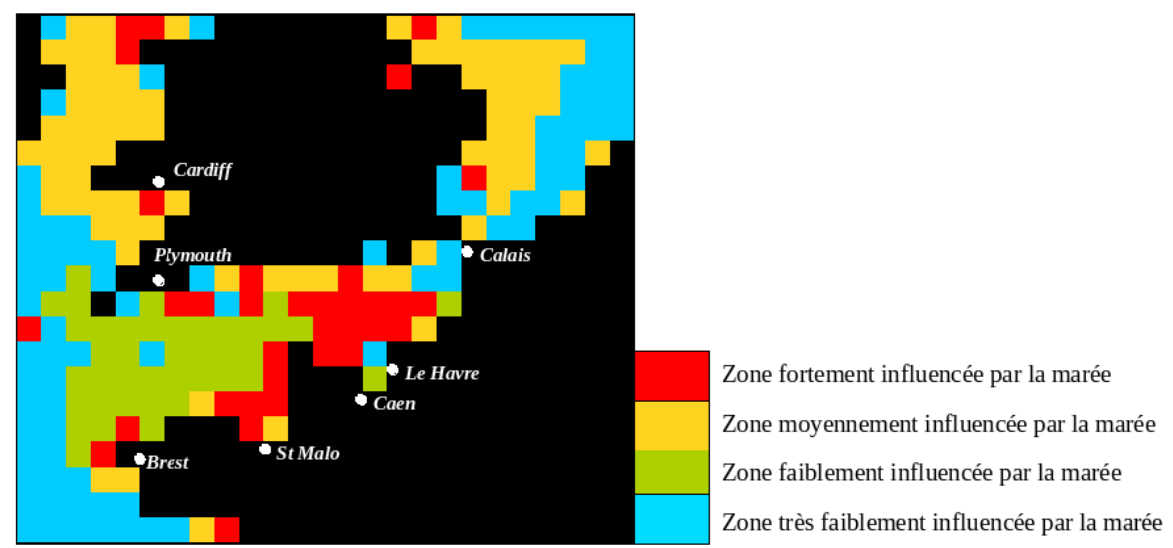

Fig. 1. Zones d'influence du cycle morte-eau/vive-eau en Manche (RIVIER et al., 2012).

\section{Matériels et méthodes}

\subsection{Modélisation numérique}

Les simulations sont effectuées à l'aide du modèle numérique hydro-sédimentaire 3D ROMS ("Régional Ocean Model System"). Le modèle hydrodynamique résout les équations primitives de Navier-Stokes sous l'hypothèse d'hydrostaticité et l'approximation de Boussinesq et calcule l'évolution temporelle des champs 3D de vitesse moyenne, d'énergie cinétique turbulente et de viscosité. Le module sédimentaire (WARNER et al., 2008) résout pour chaque classe granulométrique de diamètre sédimentaire considéré une équation d'advection-diffusion des particules en suspension dans la colonne d'eau.

ROMS est appliqué à la Manche et ses environs du 1er janvier 2007 au 31 décembre 2008 entre les longitudes $-3,3^{\circ} \mathrm{W}$ et $3^{\circ} \mathrm{W}$ et les latitudes $48,41^{\circ} \mathrm{N}$ et $51^{\circ} \mathrm{N}$ (figure 2 ). Ce domaine est étendu entre les longitudes $-4^{\circ} \mathrm{W}$ et $4,7^{\circ} \mathrm{W}$ et entre les latitudes $48,40^{\circ} \mathrm{N}$ et $52,85^{\circ} \mathrm{N}$ pour les calculs hydrodynamiques afin de disposer de conditions aux limites plus appropriées aux frontières du domaine interne (GUILLOU \& CHAPALAIN, 


\section{XII ${ }^{\text {èmes }}$ Journées Nationales Génie Côtier - Génie Civil \\ Cherbourg, 12-14 juin 2012}

2010). La paramétrisation du domaine est basée sur l'étude de GUILLOU \& CHAPALAIN (2010). Le domaine est discrétisé horizontalement en une grille de $209 \times$ 169 cellules de résolution $3 \mathrm{~km}$ et le maillage vertical est composé de 11 couches en coordonnées sigma. Les pas de temps barocline et barotrope sont respectivement de $300 \mathrm{~s}$ et de $30 \mathrm{~s}$. Le paramètre de rugosité z0 est calculé à partir de la composition du substrat. La disponibilité initiale de chaque classe est basée sur une répartition réelle hétérogène obtenue après traitement des échantillons prélevés au cours des campagnes du programme "RCP 378 Benthos de la Manche" par une méthode d'interpolation spatiale mixte AFS-krigeage (GUILLOU et al., 2010). Dans les parties étendues du domaine, z0 est fixé à 0,0035 m (GUILLOU et al., 2009).

Cinq classes de sédiments non cohésifs sont considérées allant de $25 \mu \mathrm{m}$ (silts) à $750 \mu \mathrm{m}$ (sables grossiers). La vitesse de chute et la tension critique de remise en suspension sont calculées respectivement selon les formules de SOULSBY (1997) et de SOULSBY \& WHITEHOUSE (1997). La contrainte de cisaillement totale induite par la superposition de la houle et du courant est obtenue à partir de la formulation de SOUSLBY (1997).

Le modèle est forcé par la surface libre de marée aux deux frontières ouvertes du domaine (nord et ouest) obtenue par reconstitution du niveau d'eau à partir de 10 harmoniques dérivées de la base TPXO.6 (EGBERT \& EROFEEVA, 2002). Le vent est issu de la base ALADIN (Météo-France). Les paramètres de houle (vitesse orbitale au fond, période et direction) sont extraits de simulations WW3 réalisées sur la zone d'étude dans le cadre des programmes IOWAGA et PREVIMER.

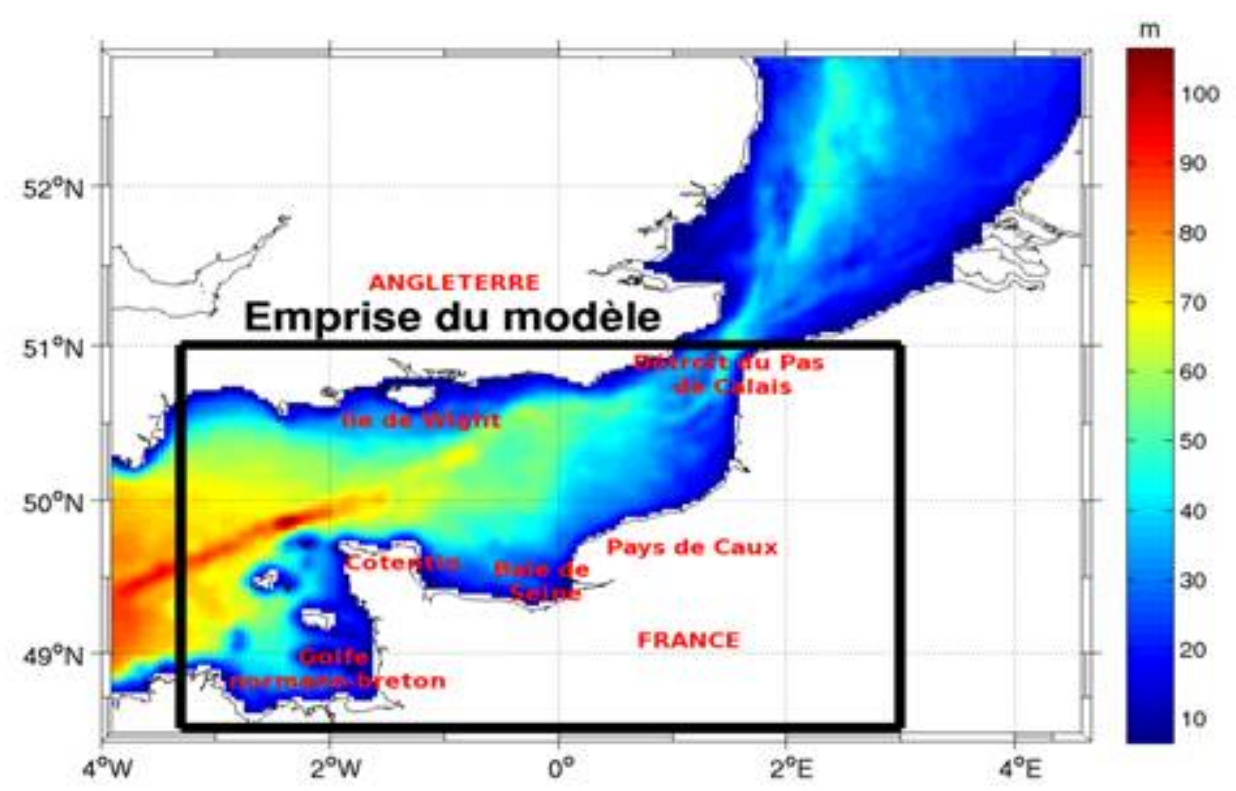

Figure 2. Bathymétrie et emprise du modèle. 


\subsection{Images satellite}

Les satellites MODIS et MERIS fournissent quotidiennement depuis 2003 des images de la couleur de l'eau sur la Manche et ses environs à une résolution spatiale de 1,2 km. Traitées par l'algorithme semi-analytique développé à l'IFREMER (GOHIN, 2011), ces données nous permettent de connaître la quantité de matières en suspension non algales qui peut être assimilée à la CSS de surface. La méthode utilise les coefficients de rétrodiffusion à 550 et $670 \mathrm{~nm}$ et fait l'hypothèse que le coefficient de rétrodiffusion (coefficient de rétrodiffusion ramené à l'unité de masses) est constant en espace et en temps. Or une particule fine rétrodiffuse plus que celles de taille supérieure. Ceci a pour conséquence que le satellite a tendance à surestimer la CSS l'hiver quand les particules en surface sont plus petites à cause de la turbulence qui casse les agrégats. L'algorithme a été testé le long des côtes françaises et a été comparé avec succès à des mesures in-situ de MES (GOHIN, 2011). La présence de nuages, particulièrement fréquents en Manche, est problématique lors de comparaisons satellite-modèle. Une sélection d'images dites "claires" a été faite sur la base du pourcentage de données dans l'emprise du modèle (figure 3). Trois images avec plus de 90\% des données disponibles et des conditions hydrodynamiques de forçages différentes ont été retenues pour les comparaisons : le 11 février, le 6 mai et le 11 octobre 2008.

Le 11 février 2008 est postérieur à une période de forte houle d'ouest (Hauteur significative (Hs) proche de $6 \mathrm{~m}$ entre le 5 et le 6 mai à l'ouest) et la marée est importante (vive-eau de coefficient 93). Le 6 mai et le 11 octobre 2008 les houles sont faibles à moyenne (Hs entre 0,5 et $2 \mathrm{~m}$ ) et la marée est respectivement forte (vive-eau de coefficient 104) et faible (coefficient de 59). Le passage du satellite n'étant pas instantané, il peut y avoir quelques minutes entre le début et la fin du passage.

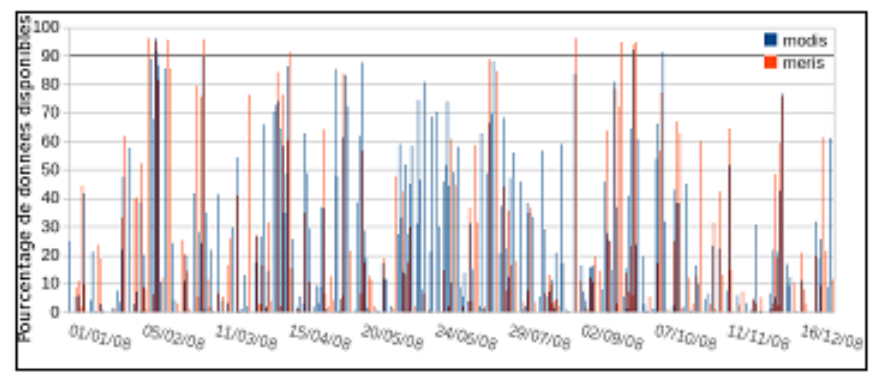

Figure 3. Pourcentage de données disponibles sur les images satellite MODIS et MERIS sur l'emprise du modèle pendant l'année 2008.

\section{Résultats et discussion}

\subsection{Comparaison satellite-modèle}

Les résultats du modèle (figure 4 , à gauche) sont comparés en surface aux données satellite (figure 4, à droite) aux trois dates. 


\section{XII ${ }^{\text {èmes }}$ Journées Nationales Génie Côtier - Génie Civil \\ Cherbourg, 12-14 juin 2012}
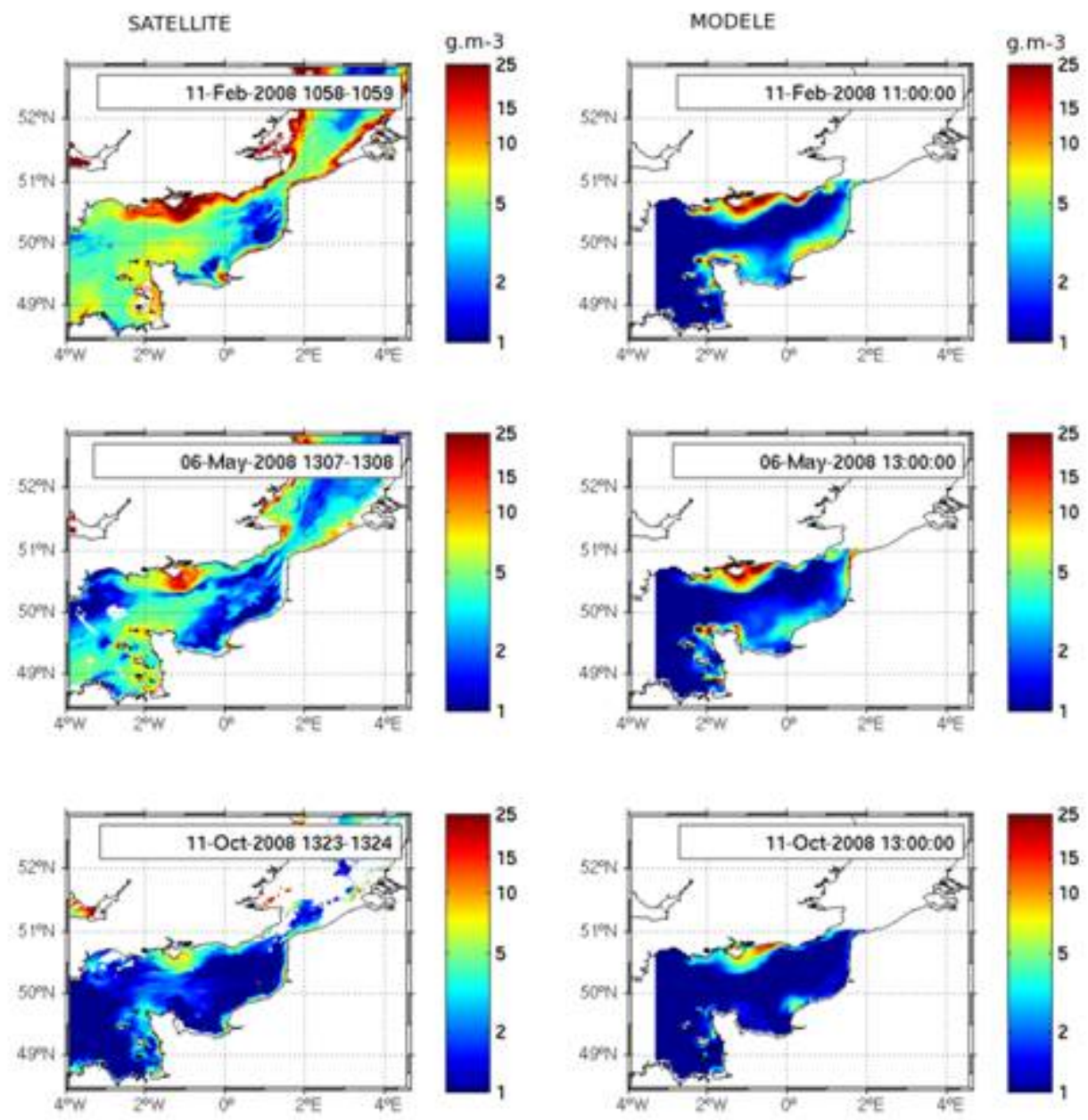

Figure 4. Comparaison satellite (gauche) - modèle (droite) de la CSS en surface (en $\mathrm{g} \mathrm{m}^{-3}$ ) les 11 février, 6 mai et 11 octobre 2008.

Cette comparaison met en évidence des variations de la répartition spatiale de la CSS de surface en fonction des conditions hydrodynamiques de marée et de houle. Les plus fortes concentrations sont ainsi observées le 11 février 2008 (figure 4-a) pour les conditions hydrodynamiques les plus importantes avec des valeurs supérieures à 4-5 mg/l en Manche centrale. Différentes zones de CSS élevées sont également révélées avec des niveaux supérieurs à $10 \mathrm{mg} / \mathrm{l}$ : autour de l'île de Wight avec un prolongement continu le long des côtes anglaises, en quelques aires localisées dans le golfe normanobreton et face à la presqu'île du Cotentin, au large du Pays-de-Caux et au niveau de l'estuaire externe de la Seine. Les niveaux de CSS observés en surface les 6 mai et 11 octobre 2008 (figures 4-b et c) sont moins importants restant limités à 2-3 mg/l en Manche centrale. Cette diminution apparaît pour des conditions de houle réduites. Les aires de CSS maximales sont restreintes à la zone proche de l'île de Wight et au niveau du golfe normano-breton avec une réduction des niveaux le 11 octobre par rapport au 6 


\section{Thème 2 - Dynamique sédimentaire}

mai. Ces zones apparaissent influencées par le cycle de morte-eau/vive-eau de la marée (Rivier et al., 2012).

Le modèle représente cette variabilité de la CSS de surface suivant les conditions hydrodynamiques (figure 4-d, e, f). Dans les trois situations, il reproduit globalement la répartition spatiale des sédiments en suspension et les zones de CSS élevée autour de l'ile de Wight, dans le golfe normano-breton et autour du Cotentin. La forme de l'aire de CSS élevée autour de l'île de Wight se prolongeant avec continuité le long des côtes anglaises est particulièrement bien reproduite en févier (figures 4-a et d). En octobre, la forme asymétrique vers l'est de cette aire de CSS élevée est obtenue autant sur l'image satellite que dans le modèle (figure 4-c et f) avec cependant une estimation du niveau plus élevé pour le modèle à l'est de l'île et la CSS issue du modèle a des valeurs proches du satellite en Manche centrale et dans le golfe normano-breton. La présence de nuages au niveau la zone méridionale du détroit du pas de Calais le 6 mai sur l'image satellite ne permet pas la comparaison dans une aire où la CSS modélisée est forte. Les principales différences entre le satellite et le modèle se situent au large du Pays de Caux où la CSS modélisée diverge horizontalement avec une extension " cross-shore » de la structure turbide adjacente à la côte et à proximité de la limite ouest du domaine où le modèle la sous-estime (figure 4-d). En Baie de Seine, le panache ne peut être reproduit en février car les apports fluviatiles ne sont pas intégrés au calcul. Les divergences observées peuvent s'expliquer à la fois par la modèle en cours de développement et qui ignore un certain nombre de processus et par les hypothèse faite dans le traitement des données satellite.

\subsection{Composition des MES en surface}

L'analyse des contributions de chaque classe granulométrique à la CSS de surface totale montre que la classe la plus fine (sédiments de diamètre inférieur à $50 \mu \mathrm{m}$ ) est quasiment la seule présente en surface dans le domaine à l'exception du golfe normanoBreton et des alentours de l'île de Wight. Dans ces deux régions, des sédiments plus grossiers (entre 100 et $500 \mu \mathrm{m}$ ) apparaissent en surface ponctuellement en espace et en temps durant le cycle de marée. Ces particules de diamètre inférieur à $50 \mu \mathrm{m}$ sont également présentes dans le golfe normano-breton et les alentours de l'île de Wight alors que le substrat y est composé principalement de sables et de cailloutis.

\subsection{Sensibilité au temps d'initialisation}

La figure 5 représente la CSS de surface le 6 mai 2008 obtenue par le modèle pour une simulation commençant les 01/01/2007 et 01/01/2008. La simulation commençant en 2007 présente des résultats plus proches de l'image satellite (figure 4 b) dans le golfe normano-breton et entre l'Ile de Wight et le Cotentin. Ceci met en avant que le temps d'initialisation a un impact sur la formation des structures de CSS. Les sédiments en suspension sortent du domaine de calcul entraînant la baisse du signal de fond de la 


\section{XII ${ }^{\text {èmes }}$ Journées Nationales Génie Côtier - Génie Civil \\ Cherbourg, 12-14 juin 2012}

CSS. Le forçage aux frontières de la CSS représente une perspective d'amélioration du modèle.

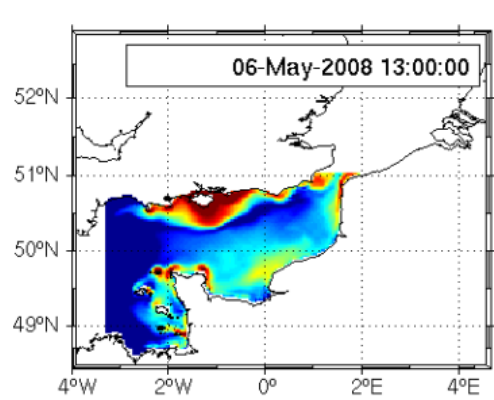

g. $m-3$

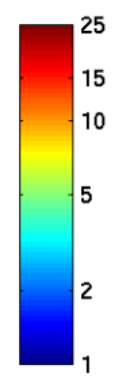

g. $m-3$

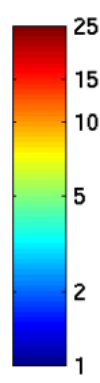

Figure 5. Comparaison entre la CSS de surface obtenue avec le modèle (en g. $\mathrm{m}^{-3}$ ) avec un départ de la simulation respectivement les 01/01/2007 (gauche) et 01/01/2008 (droite).

\section{Conclusions}

Les images satellite et le modèle montrent que la CSS évolue différemment en fonction des forçages de marée et de houle. Le modèle reproduit les structures observées par les satellites dans différentes conditions hydrodynamiques. Les particules les plus fines (diamètre inférieur à $50 \mu \mathrm{m}$ ) sont largement majoritaires en surface et contribuent principalement à la turbidité en Manche. Le modèle est en cours de développement et la prise en compte de nouveaux phénomènes tels que l'agrégation et la désagrégation va permettre d'améliorer les simulations. Ce modèle permettra de cartographier la répartition spatiale de la CSS pour divers forçages de marée et de houle et d'améliorer la qualité des modélisations biologiques opérationnelles grâce à un paramétrage plus précis de la lumière disponible dans la colonne d'eau.

\section{Remerciements}

Les auteurs remercient les programmes IOWAGA et PREVIMER pour avoir permis l'accès aux données de houle, METEO-FRANCE aux données de vent ainsi que le projet MyOcean/GMES qui met à disposition les données de couleur de l'océan issues des satellites MODIS (NASA) et MERIS (ESA). Ce travail fait parti du projet CHARM3.

\section{Références bibliographiques}

DOBRYNIN M., GAYER G., PLESKACHEVSKY A., GÜNTHER H. (2010). Effect of waves and currents on the dynamics and seasonal variations of suspended particulate matter in the North Sea. Journal of Marine Systems, n82(1-2), pp 1-20. doi:10.1016/j.jmarsys.2010.02.012 
EGBERT G.D., EROFEEVA S.Y. (2002). Efficient inverse modeling of barotropic ocean tides. J. Atmos. Oceanic Technol., nº19, pp 183-204. doi:10.1175/15200426(2002)019<0183:ЕІМОВО >2.0.CO;2

GOHIN F. (2011). Annual cycles of chlorophyll-a, non-algal suspended particulate matter and turbidity observed from space and in-situ in coastal waters. Ocean Science, nº7, pp 705-732. doi:10.5194/os-7-705-2011

GUILLOU, N., CHAPALAIN, G., LEPRÊTRE, A. (2010). Interpolation spatiale des distributions granulométriques des sédiments de fond - description théorique et applications en Manche orientale et en Mer d'Iroise. Rapport de présentation des outils, CETMEF, LGCE, $44 \mathrm{p}$.

GUILLOU N., CHAPALAIN G., THAIS L. (2009). Three-dimensional modeling of tide-induced suspended transport of seabed multicomponent sediments in the eastern English Channel. Journal of Geophysical Research, ${ }^{\circ} 114$, c07025, pp. 13, doi:10.1029/2008JC004791

GUILLOU N., CHAPALAIN G. (2010). Numerical simulation of tide-induced transport of heterogeneous sediments in the English Channel. Continental Shelf Research, n³0(7), pp 806-819.

PULS, W., DOERFFER, R., \& SUNDERMANN, J. (1994). Numerical-simulation and satellite-observations of suspended matter in the North-Sea. IEEE Journal of Oceanic Engineering, $\mathrm{n}^{\circ}$ 19(1), pp 3-9. doi:10.1109/48.289444

RIVIER A., GOHIN F., BRYÈRE P., PETUS C., GUILLOU N., CHAPALAIN G. (2012). Observed vs. predicted variability in non-algal suspended particulate matter concentration in the English Channel in relation to tides and waves. Geo-marine Letters, $\mathrm{n}^{\circ} 32$ (2), pp 139-151. doi:10.1007/s00367-011-0271-x

SOULSBY R. L. (1997). Dynamics of marine sands. Thomas Telford, H. R. Wallingford, $249 \mathrm{p}$.

SOULSBY R. L., WHITEHOUSE R.J.S.W. (1997) Threshold of sediment motion on coastal environments. Proceedings Pacific Coasts and Ports '97 Conference.

SOUZA A. J., HOLT J. T., PROCTOR R. (2007). Modelling SPM on the NW European shelf seas. Geological Society, London, Special Publications, ${ }^{\circ} 274$, pp 147-158 WARNER J.C., SHERWOOD C.R., SIGNELL R.P., HARRIS C.K., ARANGO H.G. (2008). Development of a three-dimensional, regional, coupled wave, current, and sediment-transport model. Computers \& Geosciences, n³4(10), pp 1284-1306. doi:10.1016/j.cageo.2008.02.012. 